\title{
Real Time Wireless Health Monitoring System
}

\author{
${ }^{1}$ AsadKhaliq*, ${ }^{2}$ M. AbdullahAwan,${ }^{3}$ M.UmairSaleh, ${ }^{4}$ M.Waseem Abbas, \\ HusnulMaab, ${ }^{5}$ Salman Khan \\ ${ }_{1,2,3,4,5}$ GIK Institute of Engineering Sciences and Technology,KPK,Pakistan.
}

\begin{abstract}
In this paper a Health Monitoring System is presented. The System can be easily adopted to monitor the conditions of patient or performance of an athlete in Hospital and Gymnasium respectively. Blood Oxygen level, Temperature, Heart Rate and Breath Rate are called vital signs. Human body's most basic and important functions can be described in term of vital signs. The System incorporates data acquisition of vital signs using noninvasive approach, wireless transmission of the data, results display and their interpretation using soft computing. Health of a Patient can be monitored based on vital signs. Endurance and cardiorespiratory fitness of an athlete can be described in terms of $\mathrm{VO}_{2 \max }$, Anaerobic Threshold (AT) and Total Calories Expenditure. The system is equally reliable to monitor endurance and cardiorespiratory fitness of athletes. Suitable results are achieved based on the system.
\end{abstract}

Key Words: Aerobic, Anaerobic, Endurance, Calories,Sensor, Telemonitoring, Vital signs.

\section{Introduction}

In September 1999 Business Week herald sensor technologies as the most important technologies of $21^{\text {st }}$ century [1].Recent advancements in low power wireless communications as well as improved processing and integration capacity of electronic devices have enabled to realize smart sensors for wide range of applications. The availability of low cost and power efficient sensors is playing vital role in development of new applications in many fields, including Bio-medical engineering [2]. In Bio-instrumentation, by incorporating intelligent sensors, modern telecommunication technologies and computers, it has become feasible to design telemonitoring systems to acquire, transmit, record and display physical parameters of importance [3].

In telemedicine, information and communication technologies (ICTs) are being used to overcome geographical barriers, and to increase access to healthcare opportunities. This is particularly beneficial to the groups that suffer from lack of access to health care facilities in developing countries [4].Early detection of healthcare conditions can direct physician to take necessary steps to handle or avoid unusual situation in future [5]. In telemedicine research is focused on improving quality of human life in terms of health by design, fabrication and incorporation of sensors with modern ICTs. The sensors are either in direct contact with the human body (invasive) or indirectly (noninvasive) [6]. Temperature, Heart Rate, Respiratory Rate and Oxygen Saturation in blood are considered vital parameters in patient monitoring $[7,8,9]$. These four parameters enable us to manage most of the diseases.

Endurance and cardiorespiratory fitness of an individual can be described in terms of $\mathrm{VO}_{2 \max }$, Anaerobic Threshold (AT) and Total Calories Expenditure. There are three important criterions to measure physical fitness and strength of a person.

- $\quad$ Anaerobic Threshold

- $\quad$ Maximal Oxygen Uptake

- Total Energy (calories) Expenditure

The anaerobic threshold (AT) is considered as the theoretical highest exercise level that cannot be maintained for long period of time. It has practical importance to the competitive endurance of an athlete to measure progress and plan training programs [10].Maximal aerobic power $\left(\mathrm{VO}_{2 \max }\right)$ is commonly interpreted as an index of cardiorespiratory fitness. It is the most common measurement made in physiology laboratories [11]. Unlike AT, it indicates aerobic physical fitness of individual. Total energy expenditure describes amount of energy consumed during exercise.

In this paper a telemonitoring system is presented which incorporates both hardware and software. In hardware, data acquisition processing and transmission devices are included. The physical parameters measured are Temperature, Heart Rate, Respiratory Rate and Oxygen Saturation in Blood. The system can be deployed in Hospital or Gymnasium for monitoring of disease or physical fitness respectively. 


\section{Method And Materials}

The block diagram of system is shown in Fig 2.1.The system takes up Temperature, Heart rate, Breath Rate and Blood Oxygen Level (Oxygen saturation). Sensors are connected to microcontroller. Results of sensors are sent to computer through XBee transceiver, and the computer stores data into Database. The values are displayed on Graphical User Interface (GUI).Incase of patient monitoring if there is any abnormality in physical parameters alarm is generated. System can also be used to monitor performance of athletes based on received physical parameters.

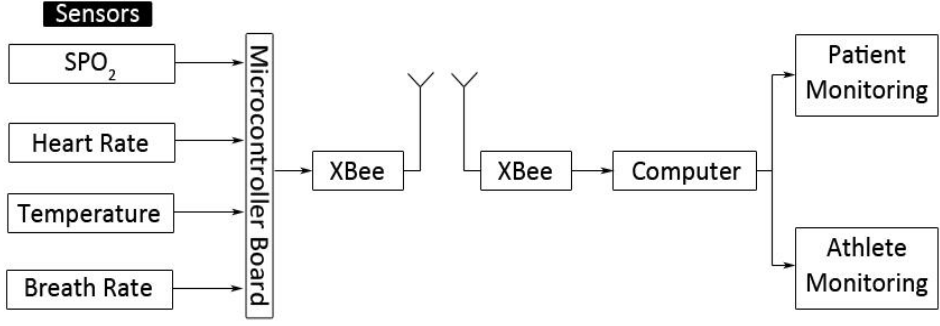

Fig. 2.1 Functional Block Diagram

\subsection{Description of Sensing System}

The system consists of four sensors Temperature sensor, Heart Rate Sensor, Breath Rate Sensor and Oxygen Saturation Sensor. Breath Rate and Temperature sensor circuitry used in the design generates analog voltage which is fed to the ADC (Analog-to-Digital) inputs of the microcontroller. The ADC input is time multiplexed and sampled at same rates. Description of sensors follows.

\subsubsection{Temperature Sensor}

For temperature sensing purpose LM35 is used which is a rapid, low-cost, integrated-circuit temperature sensor manufactured by Texas Instruments. It is a linear temperature sensor which is directly calibrated in ${ }^{\circ} \mathrm{Celsius}$ with the scaling factor of $10 \mathrm{mV} /{ }^{\circ} \mathrm{C}$. Supply voltage required by the sensor is spanned over a dynamic range of 4 to $30 \mathrm{~V}$. It has also very low self heating of less than $0.08^{\circ} \mathrm{C}$ in still air due to its very low input current drain of less than $60 \mu \mathrm{A}$. [12]. LM35 is used to measure the skin temperature of human skin at wrist. From skin temperature, the body temperature can be estimated by different methods but with the rough estimation human body temperature is $5.1^{\circ} \mathrm{C}$ higher than skin temperature. [13]. Output of sensor is connected with ADC of Arduino Uno for further processing and transmission with XBee.

\subsubsection{Heart Rate and Blood Oxygen Level Sensor}

A finger photo-plethysmograph (PPG) is a noninvasive transducer which is used to measure the heart rate and blood oxygen level of subject. The probe includes two light emitting diodes, one in visible red spectrum $(660 \mathrm{~nm})$ and other in infrared spectrum $(940 \mathrm{~nm})$ [14]. The system employs the principle of red and infra-red light absorption characteristics of oxygenated and deoxygenated hemoglobin. Oxygenated hemoglobin absorbs more infrared and less red light. Deoxygenated hemoglobin absorbs more red light and allows more infrared light to pass through. Fig2.2 shows the absorption spectra of deoxygenated hemoglobin $(\mathrm{Hb})$ and oxygenated hemoglobin $\left(\mathrm{HbO}_{2}\right)$.

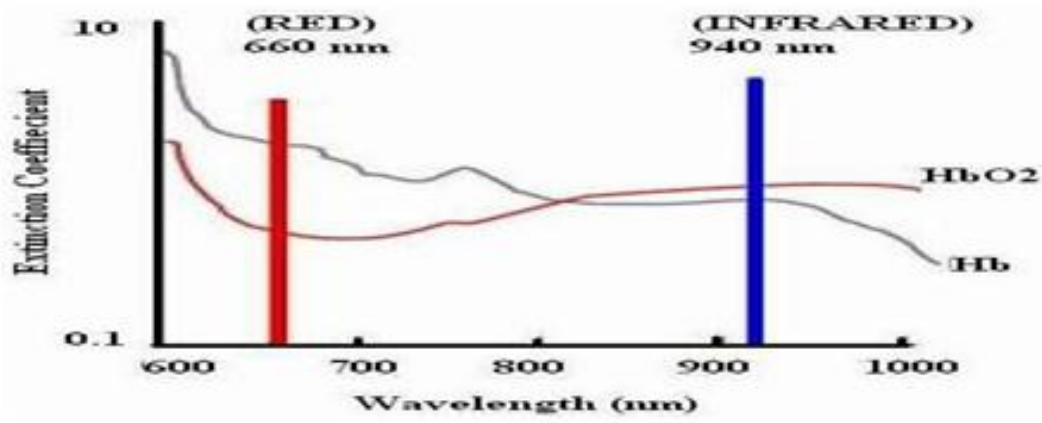

Fig. 2.2 Absorption Spectra [15]

Transmission method is used to detect light passing through the finger. In this method, the emitter and photo detector are placed opposite each other with the finger in between such that light can then pass through 
the finger as shown in Fig.2.3. An intermediate value known as Normalized ratio R [16], is calculated using the following relationship $\mathrm{R}=(\mathrm{ACR} / \mathrm{DCR}) /(\mathrm{ACIR} / \mathrm{DCIR})$

Using the value of $\mathrm{R}$, we can calculate blood oxygen level as follows $\mathrm{SPO}_{2}=110-25 * \mathrm{R}$

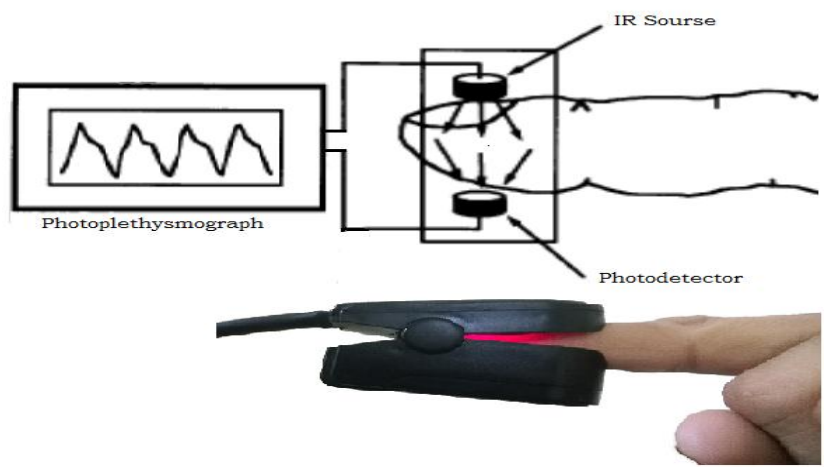

Fig. 2.3 Source and Detector Configuration

With each heart beat the heart contracts and there is a surge of arterial blood, which momentarily increases arterial blood volume across the measuring site. This results in more light absorption during the surge. If light signals received at the photo detector are looked at as a waveform, there should be peaks with each heartbeat and troughs between heartbeats. By calculating the number of peaks per fifteen seconds and multiplying the answer with four, the number of heart beats per minute (BPM) is calculated.

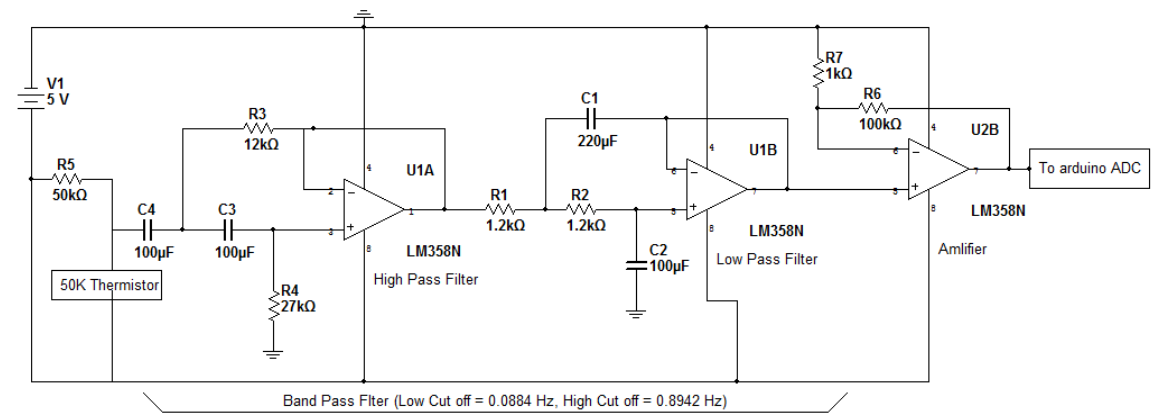

Fig. 2.4 Circuit Diagram of Breath Rate Sensor

\subsubsection{Breath Rate Sensor}

Traditional methods used for breath rate sensors include Thoracic Expansion Measurement, Thoracic Impedance Pneumography and Capnography. The main shortcoming of these methods is that they are expensive, complicated and unacceptable for long term sleeping patients [18]. Our breath rate sensor consists of a 50K negative temperature coefficient thermistor fitted in a nebulizer mask in a voltage divider configuration. Resistance of thermistor decreases during exhale due to comparatively hot air and increases during inhale. This resistance change is detected in the form of an ac signal and fed into a band pass filter to remove dc as well as other high frequency noise. [19,20,21] Circuit diagram of sensor is shown in Fig. 2.4

Band pass filter has low cutoff frequency of $0.0884 \mathrm{~Hz}$ ( 5 breaths/min) and high cutoff frequency of $0.8942 \mathrm{~Hz}(50 \mathrm{breaths} / \mathrm{min})$ and consists of a High pass filter with $\mathrm{Q}$ factor $=0.75$, Cutoff frequency $=0.0884$ $\mathrm{Hz}$ followed by Low pass filter with $\mathrm{Q}$ factor $=0.74$, Cutoff frequency $=0.8942$ in unity gain Sallen-Key topology. Output from band pass filter is amplified 100 times and fed into ADC of Arduino Uno for wireless transmission with XBee. Breath waveform is received in $\mathrm{PC}$ and breath rate is calculated with a peak detection algorithm inNILabVIEW 2010. 


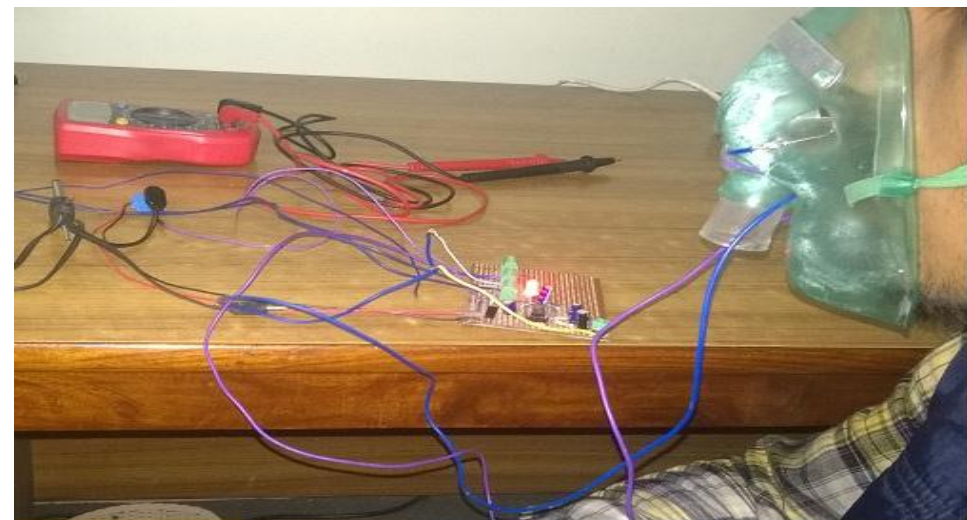

Breath Rate Sensor is being tested

\subsection{Microcontroller Interfacing}

All four sensors are interfaced with Arduino Uno, which is an open source microcontroller board based on ATmega328. It has 6 Analog inputs, 2 KB Static RAM, 1 KB EEPROM and $16 \mathrm{MHz}$ ceramic resonator. Temperature, Breath rate, Blood Oxygen level and Heart rate sensors connected to Arduino Uno analog channels. Sensor outputs are sampled at a predefined rate by an on board 10-bit ADC in a periodic manner. All the values are scaled according to the requirement and transmitted via serial communication to XBee with 9600 Bauds/s. Arduino program also associates a redundant character with each sensor value for the purpose of identification at receiving end in LabVIEW.

\subsection{Communication}

XBee 802.15.4 (Series 1) is interfaced with Arduino Uno Microcontroller Board via SparkfunXBee Shield WRL-10854.Microcontroller sends data to XBee using serial communication at 9600 Bauds/s. Both XBees, one on the sensor's side and other on computer's side, are configured to form a point to point network. $\mathrm{XBee}$ are configured using XCTU ver. 5.2.8.6 provided by Digi International Inc. Receiving end XBee is interfaced with PC's USB 2.0 port via CP2102 USB to UART Converter by Silicon Laboratories Inc. CP2102 converter creates a virtual Serial COM port, which in then is used in LabVIEW. XBee 802.15.4 (Series 1) has Indoor/Urban Range of 30m and Outdoor/RF Line-of-Sight Range of 100m.It works in the $2.4 \mathrm{GHz}$ frequency band.

\subsection{Graphical User Interface (GUI)}

Lab VIEW 2010 was used at receiver side because of its excellent GUI indicators, wide range of virtual instruments (VI's) and good real time response. Data received at COM port is separated into four streams, one for each sensor, by reading the associated code character with it. This received data isprocessed by applying different filters and algorithms to calculate final value of each parameter. Each parameter is also stored in the form of Data $\log$ in separate files. Fig. 2.5 and Fig 2.6 shows front panel of GUI for Patient and Athlete respectively.

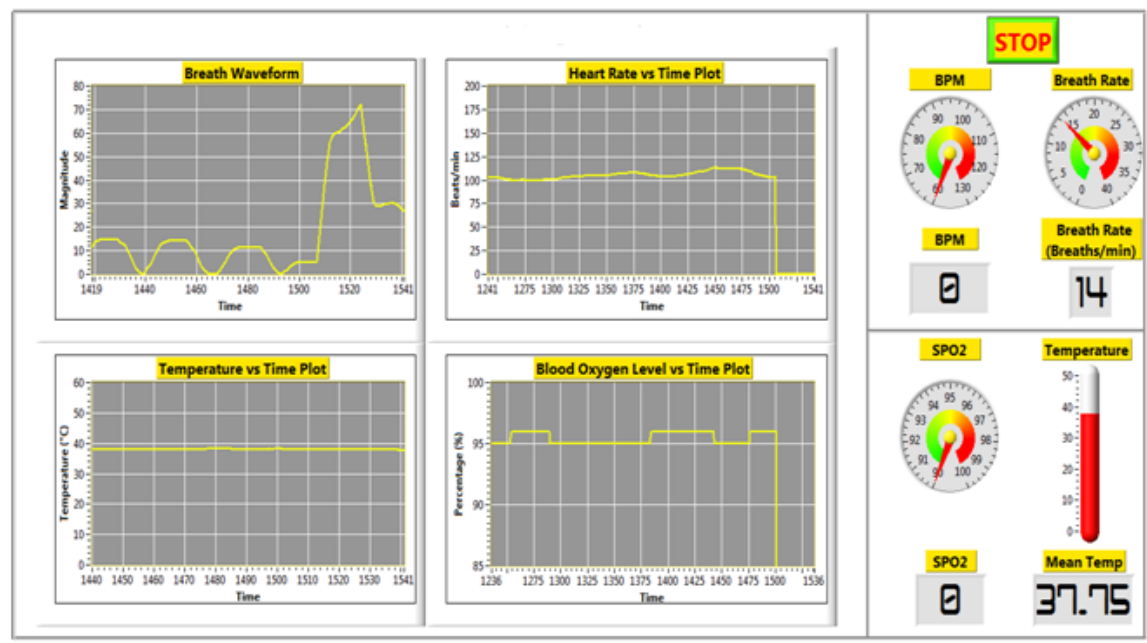

Fig. 2.5 Graphical User Interface for Patient Monitoring 


\section{Results And Discussion}

A prototype system has been developed and tested. The fabricated system can be easily adopted to monitor patients and athletes in hospital and gymnasium respectively.

\subsection{Health Monitoring in Hospital}

Vital signs (Oxygen Saturation, HeartRate, Temperature, Breath Rate) are measurements of human body's most basic and important functions. In the system, using sensors to measure four vital signs, the patient is wirelessly monitored. Results of sensors are shown in Fig 3.1 to Figure 3.5 .The graphs represent reference (actual) versus measured values of vital signs .Actual values are taken through well-established clinical methods. Figure3.5 shows overall of system.

In normal conditions Oxygen Saturation level in blood varies from 94\% to 99\% .Oxygen Saturation level below $90 \%$ leads to cardiac arrest or paralysis because of the death of certain brain and heart tissues that are very sensitive to Oxygen Saturation. ABG(Arterial Blood Gas) analyzer is being used to measure oxygen saturation.Fig.3.1 shows the comparison of oxygen saturation levels measured by the developed $\mathrm{SPO}_{2}$ sensor with respect to a $\mathrm{SPO}_{2}$ measured from $\mathrm{ABG}$ analyzer. Correlation coefficient of Oxygen Saturation Sensor is 0.9818. Results of actual and measured Heart Rate are shown in Fig3.2. Correlation coefficient between measured and actual values is 0.9912 for Heart Rate Sensor.

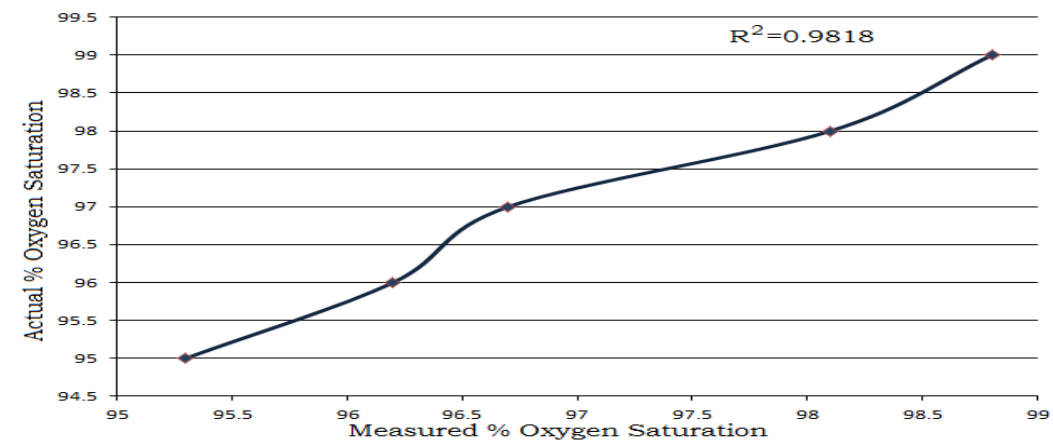

Fig 3.1 Oxygen Saturation Sensor Result

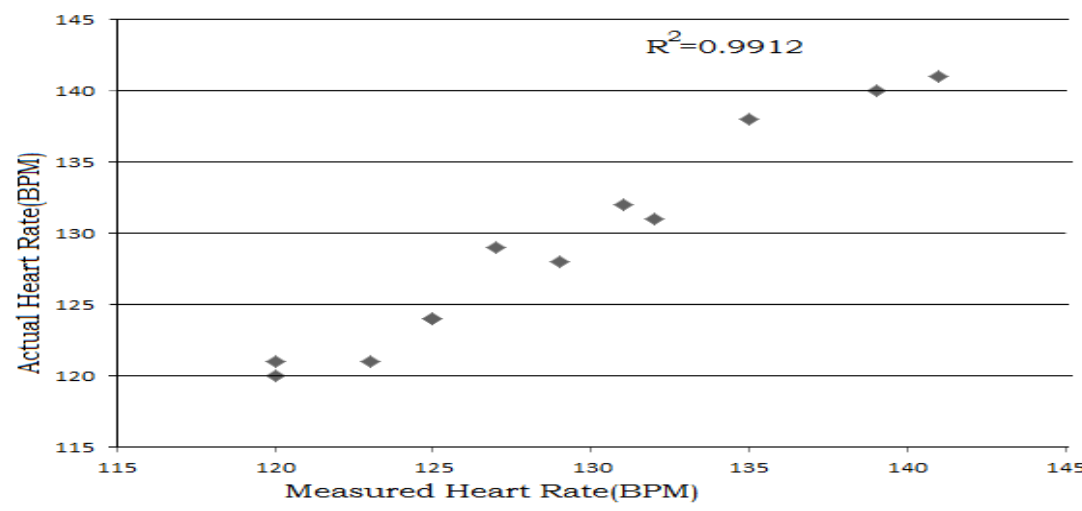

Fig 3.2 Fig 3.1 Heart Rate Sensor Result

Comparison of temperature measured by the developed temperature sensor with respect to a thermometer (actual temperature) shown in Fig 3.3.Corellation coefficient for Temperature sensor is 0.9928. Breath rate sensor is fabricated using thermistor. Slow cooling rate of thermistor limits accuracy of sensor for higher breath rates. Sensor works efficiently for up to 18 breathe/min and further increase in breath rate introduces nonlinear behavior in breathe rate sensor. Fig 3.4 shows comparison between measured and actual breath rates. 


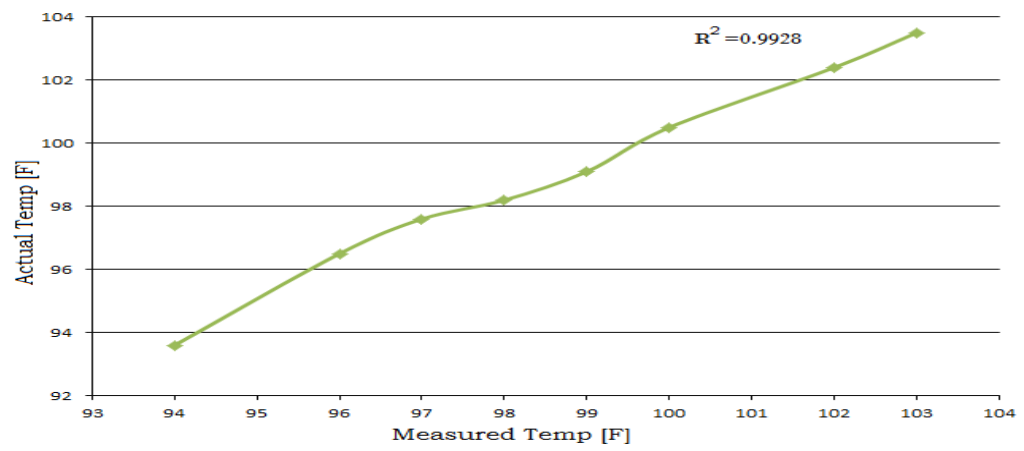

Fig 3.3 Temperature Sensor Results

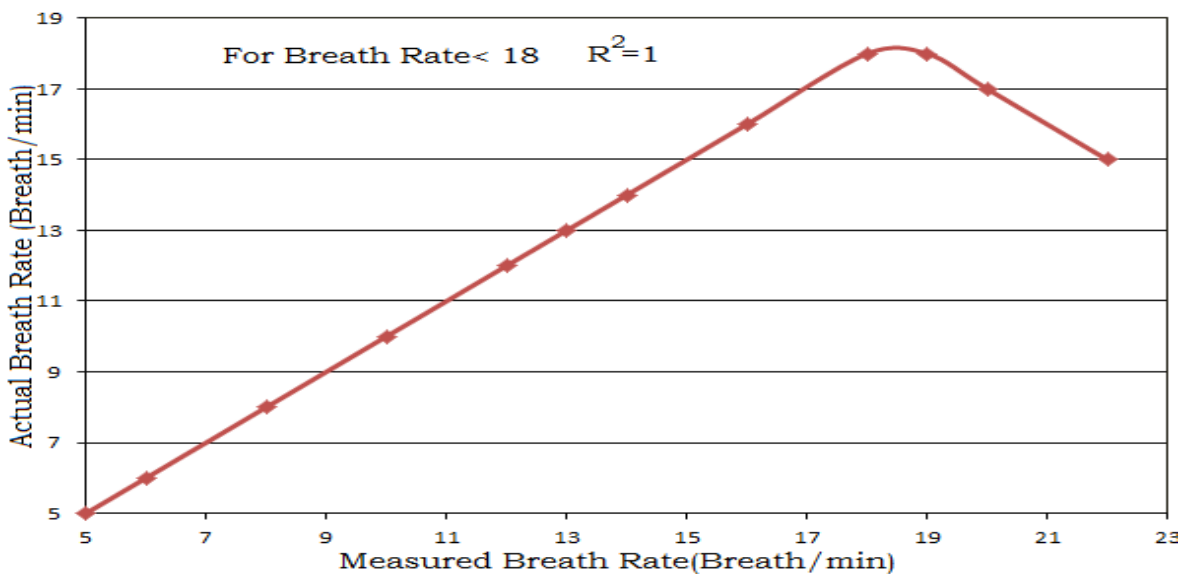

Fig 3.4 Breath Rate Sensor Result

Sample Number versus Difference (Actual-Measured) of Readings in Fig3.5 indicates results of all four sensors. Breath rate sensor has the most accurate results between $5 \mathrm{breath} / \mathrm{min}$ (least possible) to $18 \mathrm{breath} / \mathrm{min}$. Above this range breath sensor has the most inaccurate results as shown Fig 3.4.The range of accurate result for breath rate sensor can be considerably improved by incorporating high cooling rate thermistor in sensor fabrication. Heart rate sensor shows deviation from +2 to -2 as compared to actual values. It may be due to time elapse between clinical measurement and sensor measurement.

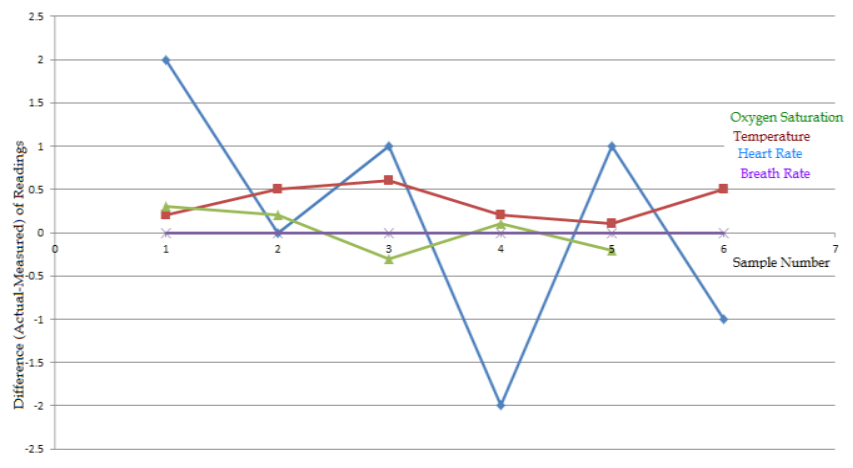

Fig 3.5 Results ofall Sensors

\subsection{Fitness Monitoring in Gymnasium}

Heart rate is direct response to any physical activity. If physical activity is intense it requires higher amount of ATPs (Adenosine Triphosphates). Availability of higher amount of oxygen is mandatory for aerobic metabolism and higherATPs production.Heart rate indicates oxygen requirements and amount of oxygen required depends on energy requirements during physical activity. Maximal oxygen uptake, anaerobic threshold, total calories expenditure are three important criterion to determine physical fitness of an individual. This section describes how to measure these three parameters using Heart Rate (HR). 


\subsubsection{Total Energy (calories) Expenditure}

Total Calories consumed during exercise can be calculated if Weight, Age, Sex, Time of Exercise and Heart Rate are given [22]. Linear regression formula can be used to calculate Total Calories Burned (TCB) during exercise [23]. New born babies have higher heart rate and it decreases with age. Maximum heart rate (MHR) islinear function of age. Maximum heart rate can be calculated if age is given [23].

TCB for Male $=((-55.0969+(0.6309 \times \mathrm{HR})+(0.1988 \times \mathrm{W})+(0.2017 \times \mathrm{A})) / 4.184) \times 60 \times \mathrm{T}$

$\mathrm{TCB}$ for Female $=((-20.4022+(0.4472 \times \mathrm{HR})-(0.1263 \times \mathrm{W})+(0.074 \times \mathrm{A})) / 4.184) \times 60 \times \mathrm{T}$

$\mathrm{HR}=$ Heart rate (in beats/minute)

$\mathrm{W}=$ Weight (in kilograms)

$\mathrm{A}=$ Age (in years)

$\mathrm{T}=$ Exercise duration time (in hours)

MHR=208-(0.7xAge)

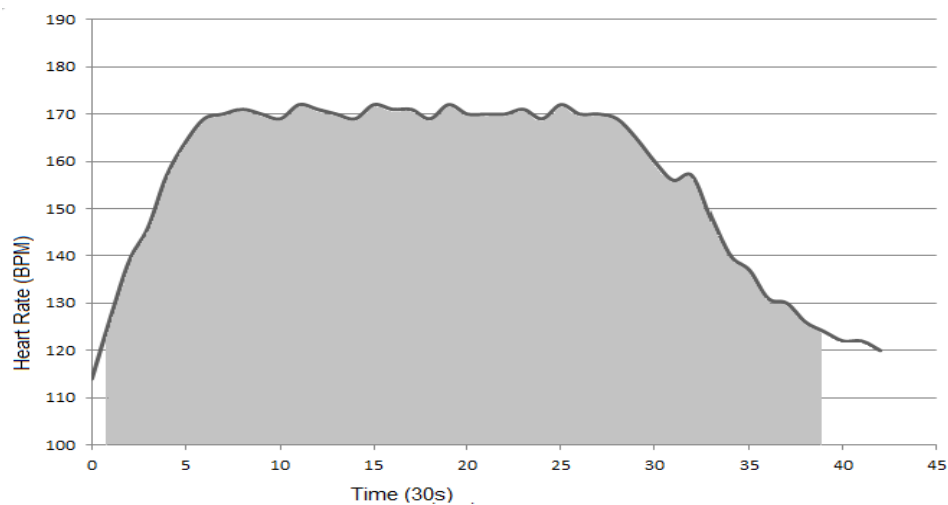

Fig 3.6 HR vs. Time Graph

In order to get reliable estimate of calories expenditure from above regression formulas, heart rate must be above 64\% of MHR[22]. Experimental data of heart rate for 23 minutes is shown in Fig 3.6.It is the data of person with 20 years of age. Shaded area below the curve represents where regression formula isvalid because Heart Rate $>64 \%$ of MHR. After each 30 seconds, monitoring system takes heart rate calculates TCB up to that interval of time.In this way using regression formula health monitoring system calculates energy expenditure in Calories during exercise.

\subsubsection{Maximal Oxygen Uptake $\left(\mathrm{VO}_{2 \max }\right)$}

$\mathrm{VO}_{2 \max }$ is maximum rate of oxygen consumption that determines capacity to perform sustainable exercise. Higher $\mathrm{VO}_{2 \max }$ indicates availability of ATPs in large amount during aerobic exercise. It is major determinant of endurance and cardiorespiratory fitness of individuals. During aerobic exercise if $\mathrm{HR}$ is known then endurance can be measured in terms of $\mathrm{VO}_{2 \max }$. If $\% \mathrm{MHR}$ (Maximum Heart Rate ) is given $\% \mathrm{VO}_{2 \max }$ can be calculated [22] by a relation given below.\% $\mathrm{VO}_{2 \max }$ for sprint and long slow distance (LSD) exercises is shown in Fig 3.7.If HR and Age is given health monitoring system can calculate $\% \mathrm{VO}_{2 \max }$ of an individual during exercise.

$\% \mathrm{VO}_{2 \max }=1.5472 \times \% \mathrm{MHR}-57.53$

$\% \mathrm{MHR}=$ Percentage of maximum heart rate

$\% \mathrm{VO}_{2 \max }=$ Percentage of $\mathrm{VO}_{2 \max }$

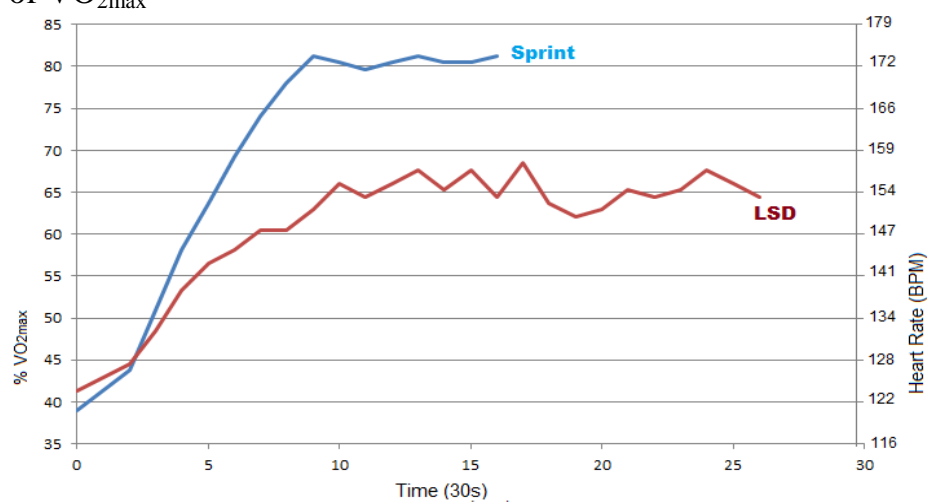

Fig 3.7 $\% \mathrm{VO}_{2 \max } \mathrm{vs}$ Time Graph 


\subsubsection{Anaerobic Threshold}

During incremental exercise a stage comes when ATPs supply from aerobic metabolism is not stuffiest. Exercise beyond this limit is not sustainable and triggers formulation of lactate acid which is by-product of anaerobic metabolism. Lactate inflection point (LIP) or AT.is the exercise intensity at which anaerobic metabolism starts. Beyond this exercise limit, both aerobic and anaerobic metabolism work together in order to supply required ATPs. Experimental data of sprint is given in Fig 3.7. Intensity of exercise for which heart rate is above $85 \%$ of MHR, starts anaerobic metabolism and defined as anaerobic threshold. During exercise, monitoring system calculates power of athlete from TCB for which heart rate is $85 \%$ to $90 \%$ of MHR.

\section{Summary}

In this paper a real time telemonitoring system for health care is presented. In order to monitor conditions of patients and performance of athletes the system can be used in hospitals and gymnasiums. The system consists of sensors (Temperature, $\mathrm{SPO}_{2}$, Heart Rate, Breath Rate) for data acquisition, data transmission devices and LabVIEW for Soft Computing and graphical user interface (GUI). Received physical parameters can be used for calculation of Anaerobic Threshold (AT), $\mathrm{VO}_{2 \max }$ and Total Calories Burned (TCB) during exercise. In fitness laboratories performance of athletes is judged with VO2max, AT and TCB. Proposed Health Monitoring System can considerably improve existing infrastructure of Hospitals and Gymnasiums. Results of vital signs monitoring are accurate and energy estimations based on the system are reliable.

\section{References}

[1]. "21 ideas for the 21st century," Business Week, pp. 78-167, Aug. 30,1999.

[2]. G. K. Lorincz, et al., "Sensor networks for emergency response: challenges and opportunities", Pervasive Computing, IEEE, vol.3, no.4, pp. 16-23, Oct.-Dec. 2004

[3]. Aleksandar C. Zoric, SinisaS.llic, "PC Based Electrocardiography \& Data Acquisition", TELSIKS, IEEE, pp 619-622, September 28- 302005 .

[4]. "Telemedicine, Opportunities and development in member states", World Health Organization, pp. 8-11, Vol.2, 2010.

[5]. U. Anliker, J. A. Ward, P. Lukowicz, G. Tröster, F. Dolveck, M. Baer, F. Keita, E. Schenker, F. Catarasi, and R. Schmid, "AMON: A Wearable multi parameter medical monitoring and alerts system", IEEE Trans. On Inf. Technol., Biomed., vol. 8, no. 4, pp. 415-427, 2004.

[6]. Y. Hao and J. Foster, "Wireless sensor networks for health monitoring applications," Physiological Meas., vol. 29, no. 11, pp. 2756, 2008.

[7]. P.Agrawal, S.P.Hingway, B.P.Dharaskar, "Innovative Approach for Wireless Health Monitoring System Using Client-Server Architecture", IJETT - Vol.4, no.5-, pp.1343-1348,May 2013

[8]. Y. S. Satyanarayan, Y. R. Satyanarayan, D. H. Desai, "Intelligent Wireless Emergency Alert System for Patient Monitoring using AT89S52 Microcontroller", IJAREEIE, Vol. 2, no. 4,pp.1224-1230, April 2013

[9]. DaomingZhang; Celler, B., "Monitoring physiological signals duringrunning exercise," Proc. of23rdIEEEAnnualIntl. Conf., Vol. 4, pp.3332- 3335,2001.

[10]. D.G. Carey, L. A. Schwarz, G.J. Pliego ,R. L. Raymond, "Respiratory Rate is a valid and Reliable Marker for the Anaerobic Threshold: Implications for Measuring Changes in Fitness", Journal of Sports Science and Medicine, Vol.4, pp.482-488, 2005.

[11]. L.B.Rowell, "Human Cardiovascular Adjustment to Exercise and Thermal Stress",Physiol.Rev, Vol.54, pp.75-103, 1974.

[12]. A.A.Tahat, "Body Temperature and Electrocardiogram Monitoring Using an SMS-Based Telemedicine System", In Proc,Wireless Pervasive Computing ISWPC,Feb 2009, pp.1-5.

[13]. R. Lenhardt, D. I. Sessler, "Estimation of mean-body temperature from mean-skin and core temperature," Anesthesiology, vol. 105, no. 6, pp. 1117-1121, Dec. 2006.

[14]. D. I.Shin, S.J.Huh , P.J.Pak, "Patient Monitoring System using Sensor Network Based on the ZigBee Radio" In Proc ,Information Technology Applications in Biomedicine, IEEE, Nov 2007, pp.313-315.

[15]. J. Hart, S.Iddir, R.Mahar,N.Thonakkaraparayil, "P.o.Pro Wireless Reflectance Pulse Oximeter 2" Internet www.bme.uconn.edu [April ,2014].

[16]. Y.Liu and R. Sahandi, "Zigbee Network for Remote Patient Monitoring on General Hospital Wards" in ProcInformation,Communication,AutomationTechnologies, IEEE, Oct 2009 ,pp.1-7.

[17]. G.Di, X.Tang, W.Liu, “A Reectance Pulse Oxime-ter Design Using the MSP430OF149”,In Proc, International Conference on Complex Medical Engineering IEEE/ICME, May 2007, pp.1081-1084,

[18]. E.Jovanov, D.Raskovic, R.Hormigo, “Thermistor-based Breathing Sensor for Circadian Rhythm Evaluation” Biomedical sciences instrumentation, vol.37,pp.493-497,feb 2001

[19]. J.S.A.Mary, R.Divya, "Monitoring the Respiratory System using Temperature Sensor "International Journal of Science and Engineering Application"s, Volume 2, Issue 5, 2013,

[20]. ArchitaAgnihotri , "Human Body Respiration Measurement Using Digital Temperature Sensor with I2c Interface" International Journal of Scientific and Research Publications, Volume 3, Issue 3, March 2013

[21]. M.Gupta, H.Qudsi, "Low-Cost, Thermistor Based Respiration Monitor" In Pros Bioengineering Conference (NEBEC), 2013, pp. $287-288$

[22]. L.R. Keytel, J.H. Goedecke, T.D. Noakes, H. Hiiloskorpi, R. Laukkanen, E.V. Lambert, "Prediction of energy expenditure from heart rate monitoring during submaximal exercise", Journal of Sports Sciences, 2005.

[23]. D.P.Swain K.S. Abernathy KS, C.S.Smith S.J. Lee,S.A. Bunn, "Target heart rates for the development of cardiorespiratory fitness", Med Sci Sports Exerc.Vol.20,no.1,pp.112-116 January 1994.

[24]. H.Tanaka. K.D. Monhan.,D.G. Seals., “Age-predicted maximal heart rate revisited”, Am CollCardiol , Vol.26, pp.153-156,2001. 